\title{
Avaliação de Acessibilidade em Documentos Digitais: Uma Aplicação no Contexto Educacional
}

\author{
Cristina Paludo Santos, Cristiane Ellwanger, Maikel Losekann
}

\author{
Universidade Regional Integrada do Alto Uruguai e das Missões (URI) \\ 98.802-470 - Santo Ângelo - RS - Brasil \\ paludo@santoangelo.uri.br, cristianeellwanger@gmail.com, \\ maikellosekann@hotmail.com
}

\begin{abstract}
This paper presents a computational solution that aims to guide users in production / adaptation of digital documents in order to make them accessible. The Accessibility Evaluator in Digital Documents - AvaDoc implements the guiding principles for creating accessible documents serving as a guide, transmitting recommendations referred to in the guidelines WCAG 2.0, for operational purposes and applied to documents generated in the most common formats. The use of the AvaDoc in the educational context is related to the creation of pedagogical materials accessible, providing subsidies for the production of documents that can be used to the greatest extent possible, by all independent learners of your profile.
\end{abstract}

Resumo. Este artigo apresenta uma solução computacional, referenciado como AvADoc -Avaliador de Acessibilidade em Documentos Digitais, o qual tem como objetivo orientar os utilizadores na produção/adaptação de documentos digitais com vistas a torná-los acessíveis. Para tanto, fornece direcionamentos para a criação de documentos acessíveis, seguindo as recomendações referenciadas nas diretrizes WCAG 2.0. Seu uso no contexto educacional está atrelado à produção de materiais pedagógicos acessíveis, promovendo subsídios para a disponibilização de documentos que possam ser utilizados, na maior extensão possível, por todos os aprendizes independentes do seu perfil.

\section{Introdução}

Dentre os grandes desafios científicos da computação apontados pela SBC para a década (2006-2016) está o "Acesso participativo e universal do cidadão brasileiro ao conhecimento" (SBC 2006). A ênfase na concepção de documentos digitais acessíveis é uma característica deste desafio visto a necessidade de extensão das informações e/ou conhecimento ao cidadão comum, em sua diversidade, respeitando suas diferenças.

Esse desafio ganha novas proporções no cenário educacional pela sua relevância no apoio ao processo de ensino e de aprendizagem que atendam às especificidades de todos os aprendizes. E, em se tratando do contexto educacional brasileiro, há que se considerar que a inclusão de aprendizes com deficiência na sala de aula do ensino regular tem desafiado educadores a utilizar práticas pedagógicas diferenciadas o que pressupõe, inclusive, a produção de materiais digitais acessíveis que possam ser utilizados, na maior extensão possível, por todos os aprendizes independentes do seu perfil [Defendi 2011]. 
O problema concerne, no entanto, à falta de conhecimentos referentes aos parâmetros de acessibilidade a considerar na produção de materiais por parte de muitos educadores. Por um lado há preocupação no atendimento das necessidades dos "novos públicos", mas, por outro, falta-lhes um suporte adequado para que possam criar e/ou adaptar seus materiais didáticos de forma a disponibilizá-los a todos os aprendizes.

Neste sentido, a área da computação agrega várias contribuições, visto que provê conceitos e métodos que permitem maximizar o processo de produção de documentos digitais, quebrando as barreiras da acessibilidade, quando empregados de forma coerente. Algumas iniciativas já existem neste sentido como, por exemplo, da empresa Microsoft que provê recursos de acessibilidade em seus produtos [Office 2013]. No entanto, o uso de tais recursos não é uma tarefa trivial e gera dúvidas e dificuldades para pessoas com pouca familiaridade no uso dos aplicativos. Além desta, outras propostas enfatizam a avaliação de acessibilidade em sites Web, como o daSilva [DaSilva 2013], ASES - Avaliador e Simulador de Acessibilidade de Sítios [ASES 2014] e HERA [HERA 2013]. No entanto, eles não tratam de particularidades existentes em documentos digitais gerados pelos aplicativos mais comuns.

Nesta perspectiva enquadra-se o AvaDoc - uma solução computacional que visa preencher lacunas ainda existentes no processo de abranger acessibilidade em documentos digitais. Tal solução pode ser categorizada como um avaliador de acessibilidade para documentos digitais e se comporta como uma ferramenta de apoio que orienta a inclusão de acessibilidade em conteúdos digitais produzidos pelos aplicativos mais comumente usados como, por exemplo, os do Microsoft Office (Word, PowerPoint, Excel) e do Open-office (Writer, Impress, Calc), disponibilizados nos formatos DOC/ODT, PPT/ODP e XLS/ODS ou PDF.

Uma visão geral do AvADoc é apresentada nas seções subsequentes, estruturadas da seguinte forma: a Seção 2 descreve as regras de acessibilidade consideradas pelo AvADoc para a produção dos documentos digitais, bem como os principais conceitos que embasaram a pesquisa; na Seção 3 é apresentado o protótipo do avaliador em desenvolvimento, incluindo as principais interfaces da ferramenta e os resultados obtidos a partir das avaliações realizadas até o momento e, por fim, a Seção 4 apresenta conclusões e direcionamentos para trabalhos futuros.

\section{Princípios Norteadores do AvaDOC}

O conceito de acessibilidade pode ser denominado como "flexibilidade proporcionada para o acesso à informação e à interação, de maneira que usuários com diferentes necessidades possam acessar e usar esses sistemas" [Melo e Baranauskas 2006]. Entende-se assim, a partir desta denominação, que a acessibilidade não se destina apenas a pessoas com deficiência, mas têm em vista todos os utilizadores incluindo àqueles com incapacidades pontuais ou permanentes ou até com problemas relacionados com a tecnologia ou com o meio.

No contexto da aprendizagem, a informação e o conhecimento devem ser recebidos por todos os aprendizes, independentemente do formato onde o mesmo é transmitido. Assim, a acessibilidade em documentos digitais permite uma harmonia bem sucedida entre informação e comunicação com relação às necessidades e preferências individuais de um usuário permitindo que o mesmo interaja e perceba o conteúdo intelectual da informação ou comunicação. $O$ conceito inclui ainda a 
capacidade de usar qualquer tecnologia assistiva ou dispositivo envolvido em seu contexto que atenda aos padrões convenientemente escolhidos [Cusin e Vidotti 2013].

Com base nestas considerações, três razões fundamentais norteiam a produção de conteúdos acessíveis no âmbito do AvADoc que são: (1) Possibilitar o acesso aos conteúdos a todas as pessoas, incluindo as pessoas com deficiência, independentemente do tipo de incapacidade; (2) Facilitar a navegação e compreensão dos conteúdos ao maior número de pessoas, dando maior visibilidade aos seus conteúdos e, (3) A acessibilidade é um valor acrescentado, beneficiando todos os aprendizes/utilizadores.

A Figura 1 apresenta um esquema conceitual do processo de verificação da acessibilidade em documentos digitais no avaliador proposto.

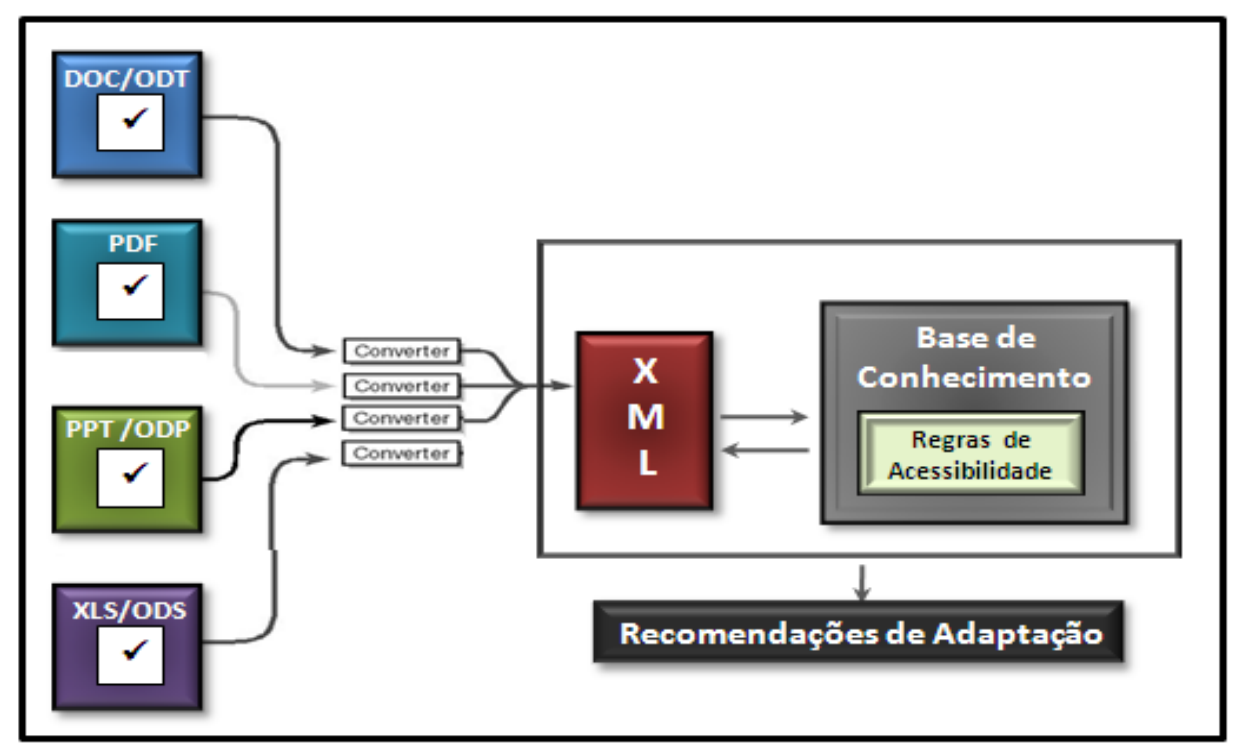

Figura 1. Esquema conceitual do processo de verificação da acessibilidade AvADoc

O processo inicia-se com a entrada, pelo utilizador, de um documento disponível em um dos formatos representados na Figura 1. Este documento é então convertido para XML por meio do uso de conversores específicos para cada formato como, por exemplo, o Databeam. Word Component [Conversor 2013] que converte documentos do formato DOC para XML.

Uma vez convertido o documento de origem é feita uma varredura no conteúdo do arquivo XML em busca de tags específicas como, por exemplo, a tag <w:pict> que identifica a presença de imagens no texto. Para cada tag encontrada é feita uma comparação com as regras de acessibilidade armazenadas. A Figura 2 apresenta um trecho de código XML em que é possível verificar a presença de tags que identificam uma imagem.

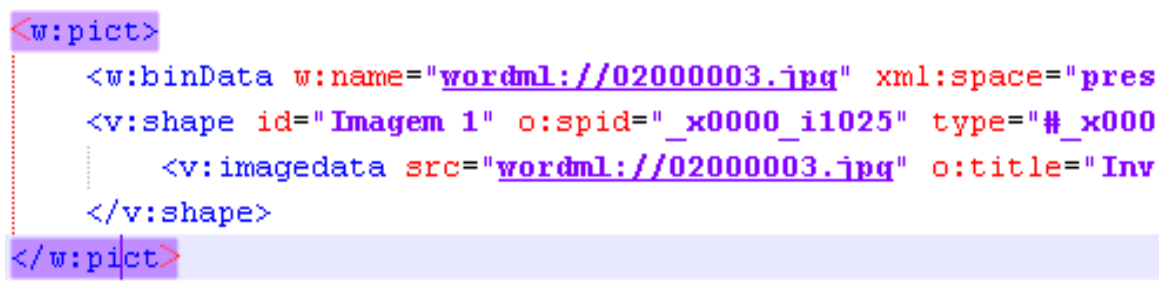

Figura 2. Trecho de código XML que identifica uma imagem 
Uma vez completado o processo de verificação do documento e comparação com as regras de acessibilidade, um relatório é emitido ao utilizador contendo informações sobre as partes do texto que deverão ser alteradas com vistas a contemplar as condições de acessibilidades necessárias.

Assim, para atender aos objetivos da aplicação proposta, foram consideradas as diretrizes WCAG 2.0 [WCAG 2014] que sugerem técnicas para produzir conteúdo acessíveis ao maior número de pessoas quer estas tenham algum tipo de deficiência ou não. Algumas das diretrizes são transversais a todos os formatos de conteúdo, apesar deste trabalho focar apenas nos formatos mais comuns já referidos anteriormente.

Dentre as recomendações gerais que compõem o AvaDoc destacam-se às provenientes da WCAG2ICT que orienta sobre a aplicação da WGAG 2.0 para Tecnologias da Informação e Comunicação não-Web. A diretriz 1.1 refere-se a "Alternativas de Texto" e tem como recomendação fornecer alternativas em texto para qualquer conteúdo não textual para que ele possa ser transformado em outras formas que as pessoas com necessidades especiais possam compreender. Em tal diretriz se enquadra o recurso de "Texto Alternativo", em que os leitores de tela apresentam o texto no lugar da imagem. Já, a diretriz 1.4 denominada "Distinguível" recomenda tornar mais fácil para os usuários a ver e ouvir o conteúdo, o que inclui a separação do primeiro plano de fundo, como o uso de uma relação de contraste de, no mínimo, 4.5:1. Nesta diretriz também se enquadram o alinhamento do texto, preferivelmente alinhado à esquerda, e também tamanho de fonte mínimo (mínimo 11 ou 12 para texto e 22 ou 24 para apresentação) incluindo o tipo de fonte que inclui o não uso de fontes estilizadas, para isso recomenda-se os tipos Arial, Tahoma ou Verdana. $\mathrm{O}$ espaçamento entre linhas, recomendado deve ser, no mínimo, de um espaço e meio.

Com base em tais diretrizes, foram definidas as seguintes regras no escopo do AvaDoc:

- Largura da página: A largura da página não deve ter mais de 80 caracteres visto que linhas de texto muito extensas dificultam a fixação da posição no texto e a passagem para a linha seguinte por parte de pessoas com algumas incapacidades de leitura ou de visão.

- Alinhamento: Os textos devem estar alinhados à esquerda. Texto justificado deve ser evitado, pois assume diferentes espaçamentos entre palavras, dificultando a leitura a pessoas com dificuldades cognitivas.

- Tipo de letra: Uso de letras do tipo Verdana, Arial ou Tahoma são as mais indicadas. $\mathrm{O}$ uso de letras serifadas ou estilizadas não é apropriado, pois as mesmas dificultam a leitura a pessoas com baixa visão, dislexia ou com incapacidades cognitivas.

- Tamanho da letra: O tamanho da letra deve ser adequado com a finalidade do documento e/ou do espaço físico onde é apresentado. Por exemplo, para o corpo de texto recomenda-se fonte de tamanho 12 ou 11. Já para apresentações sugere-se fonte no tamanho 24 ou 22. Títulos e subtítulos devem ter um tamanho igual ou superior ao corpo do texto.

- Espaçamentos: Entrelinhas o espaçamento mínimo deve ser de um espaço e meio $(1,5)$. Já, entre parágrafos, no mínimo 1,5 vezes maior do que o espaçamento 
entrelinhas. Ou seja, deve ser acrescentado 1 espaçamento antes e depois do parágrafo. Isso se deve pelo fato de que pessoas com incapacidades cognitivas têm dificuldade em acompanhar textos com linhas próximas umas das outras.

- Ortografia: O documento não deve conter erros ortográficos, pois os mesmos provocam uma leitura incorreta pelos leitores de ecrã, podendo induzir ao erro.

- Fundo: Não devem ser utilizadas marcas d'água ou imagens de fundo, pois dificultam a leitura e podem alterar o sentido do texto.

- Contraste: O contraste entre as cores do texto e fundo deve ter uma relação mínima de 4,5:1, sendo o ideal de 7:1. Contrastes muito baixos entre a cor do texto e a cor do fundo dificultam a leitura e compreensão da informação a pessoas com incapacidades visuais e cognitivas.

- Hiperlinks: A clareza e singularidade do texto de um hiperlink facilitam a sua identificação. Os leitores de tela facultam teclas de atalho para listar e saltar diretamente para os hiperlinks existentes no conteúdo. Assim, os textos devem ser claros e únicos. Não se deve utilizar a mesma palavra para diferentes hiperligações como, por exemplo, botões com a palavra "voltar", mas sim "voltar para a página 1" ou "voltar para a página 2 ".

- Resumo de gráficos: Informações sobre a estrutura e conteúdo de tabelas e gráficos pode evitar transposição de alguma célula, além de ajudar a compreender o conteúdo. Assim, sugere-se anteceder gráficos e tabelas com informações sobre a sua organização e um resumo do conteúdo.

A implementação destas recomendações evita más interpretações da informação ou uma perda excessiva de tempo na procura de soluções para transpor dificuldades [W3C 2014]. Além das diretrizes gerais acima descritas, também são consideradas as regras particulares aos vários formatos de documentos [Falkofske 2008][Turró 2008][Salton 2014]. Mais especificamente no que se refere às diretrizes relacionadas ao formato de texto estão sendo consideradas:

- Não utilizar os automatismos dos editores, tais como alíneas e numeração, pois os mesmos não são lidos pelos leitores de tela;

- Uma descrição textual de uma imagem, conhecida como texto alternativo, deve ser adicionada a todas as imagens. Os leitores de tela e as linhas Braille reconhecem o texto alternativo e apresentam o texto no lugar da imagem;

- As imagens presentes no texto devem conter uma legenda, situando-a no texto;

- As imagens de cabeçalho devem conter em seu texto alternativo que as mesmas pertencem ao cabeçalho e,

- O texto deve ser disponibilizado em apenas uma coluna, visto que alguns leitores de tela leem a primeira linha de cada coluna em vez de verticalmente ao longo de uma coluna de cada vez.

No contexto do AvADoc, tais diretrizes são armazenadas em uma base de conhecimento, formada por regras de produção, as quais delineiam os preceitos acima expostos, conforme demonstrado na Figura 3. 


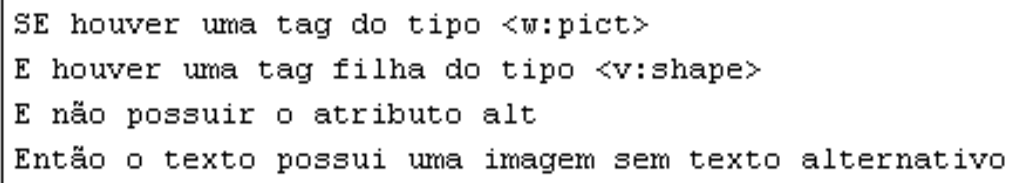

Figura 3. Regra de acessibilidade associada ao AvADoc

A regra apresentada verifica a presença ou não de texto alternativo associado a uma imagem. No total foram implementadas quinze (15) regras para representar as diretrizes consideradas nesta primeira fase de desenvolvimento da ferramenta. Novas regras estão sendo formuladas com vistas a contemplar diretrizes específicas para outros formatos de documentos como, planilhas eletrônicas e apresentações.

Cabe destacar que as alterações sugeridas são feitas pelo utilizador no aplicativo em que o documento original é criado, ou seja, a ferramenta não dispõe de ferramentas de autoria própria, pois o intuito da proposta do AvADoc é provê subsídios necessários para auxiliar utilizadores leigos na produção de documentos digitais que contemplem as diretrizes de acessibilidade.

\section{Protótipo Desenvolvido}

O AvADoc está sendo desenvolvido na linguagem de programação JAVA a fim de prover uma maior portabilidade, pois tem suas aplicações executadas por uma máquina virtual [Deitel 2003]. A Figura 4 apresenta a interface principal da ferramenta que permite ao utilizador inserir o documento a ser verificado.

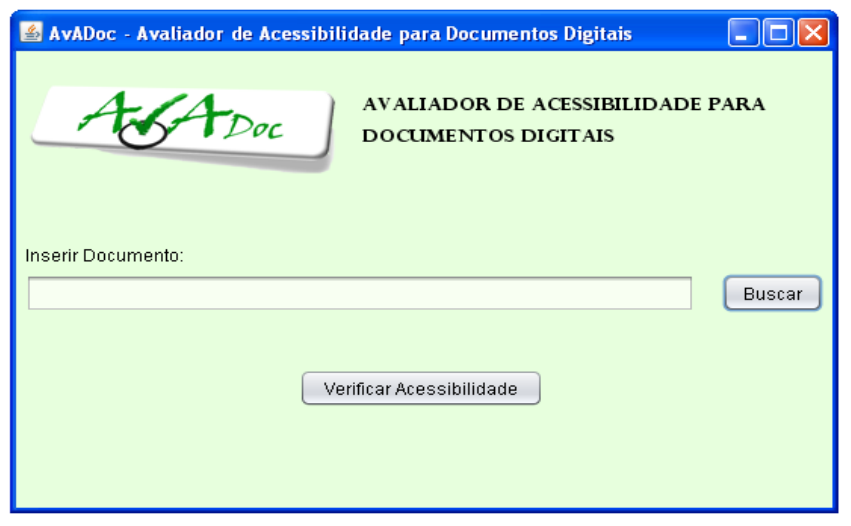

Figura 4. Tela inicial do AvADoc

Conforme demonstra o esquema conceitual da Figura 2, depois de disponibilizado o documento, o AvADoc o converte para um arquivo XML e executa a análise do documento buscando verificar se as regras de acessibilidade previstas pela ferramenta são atendidas ou não. A análise resulta em um relatório com recomendações quanto às readequações necessárias para tornar o documento acessível. A Figura 5 apresenta um exemplo de relatório gerado pela ferramenta.

O relatório segue uma estrutura bem definida com vistas a facilitar a sua leitura e entendimento pelo utilizador. Com isso o AvADoc provê recursos que permitem aos utilizadores, incluindo aqueles com nenhum conhecimento sobre as normas que regem a acessibilidade em documentos digitais, a concepção de documentos acessíveis. 


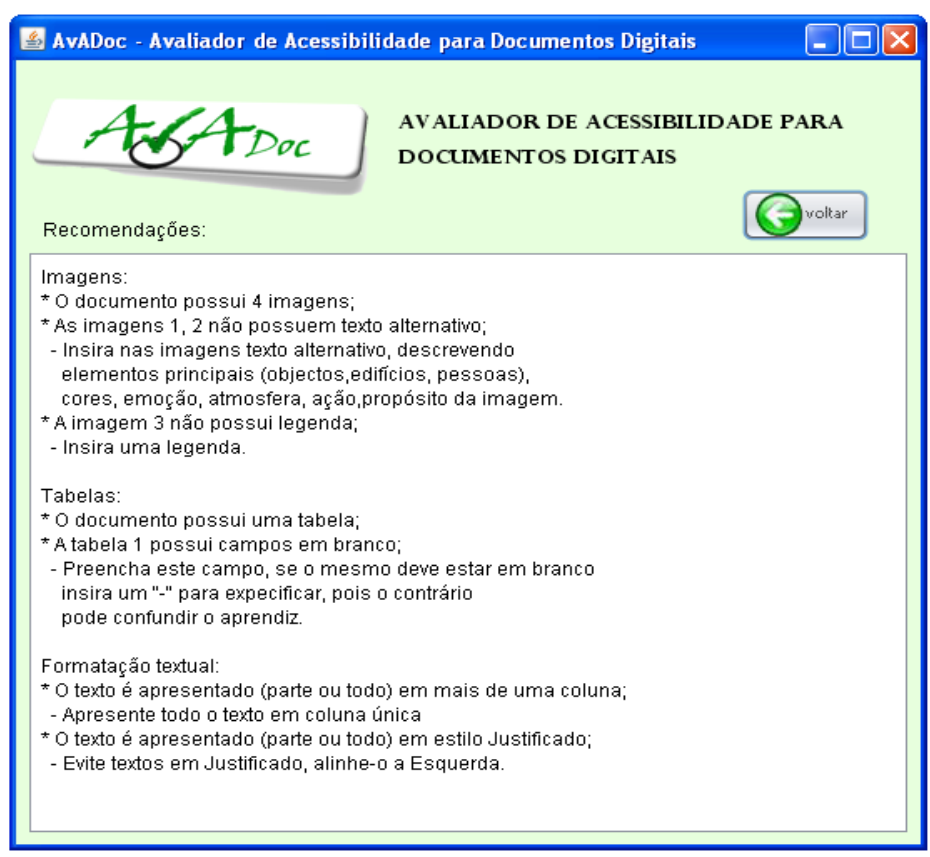

Figura 5. Relatório gerado pelo AvADoc

O AvADoc foi utilizado por educadores de uma escola de ensino fundamental que possui aprendizes com deficiência. Isso permitiu a validação do grau de usabilidade da ferramenta e, sobretudo, das recomendações geradas pelo avaliador. A partir das recomendações geradas, os educadores foram instruídos para que realizassem todas as adequações sugeridas nos materiais pedagógicos que passaram pelo processo de verificação. Uma vez executadas as modificações necessárias, a acessibilidade dos documentos gerados foi testada com os leitores JAWS R.11/R.12 e NVDA R.2010.1.

Para subsidiar o processo avaliativo do AvaDoc foram definidos critérios que permitem averiguar a adequação da ferramenta quando utilizada por educadores e, também, verificar o quanto os documentos modificados a partir das recomendações provenientes da ferramenta atendem aos princípios de acessibilidade. Dentre os critérios considerados na avaliação realizada junto a educadores destacam-se: (a) facilidade no uso da ferramenta; (b) clareza das recomendações; (c) facilidade na inclusão de acessibilidade com base nas recomendações e, (d) impacto dos materiais produzidos no processo de ensino e de aprendizagem. Já, considerando a avaliação realizada junto ao aprendizes, os aspectos que foram analisados incluem: (a) percepção de conteúdos não textuais; (b) facilidade de acesso a todas as partes do documento e, (c) presença de situação indesejável.

O processo contou com a colaboração de 3 educadores que foram instruídos a selecionar materiais digitais que utilizam em suas aulas e aplicá-los à ferramenta. Para cada um dos documentos processados pelo AvaDoc deveriam ser realizadas as alterações recomendadas de forma a torná-lo acessível. A Figura 6 apresenta o resultado da avaliação considerando questões pertinentes aos critérios previamente definidos. 


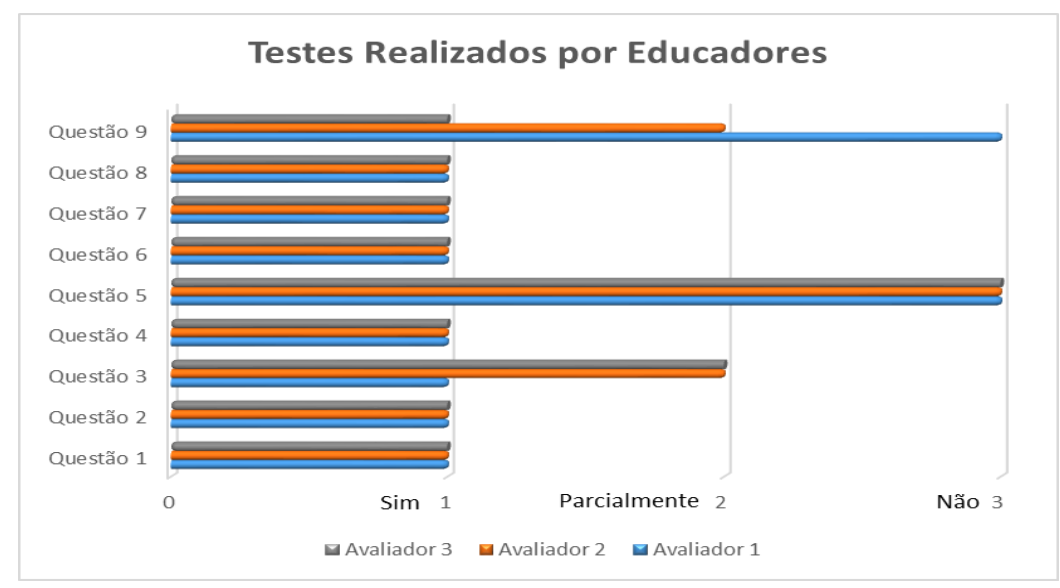

Figura 6. Avaliação do AvaDoc realizada pelos educadores

A partir dos resultados obtidos constata-se que todos os educadores apresentaram facilidade na interação com a ferramenta (questão 7), na importação de documentos (questão 1) e no entendimento a respeito das recomendações provenientes do processo (questão 2). Dos 3 participantes, 2 deles realizaram parcialmente as alterações no documento original (questão 3) e relataram que o documento final apresentou partes inacessíveis aos usuários finais (questão 9). Visto que todos relataram que as dificuldades encontradas não ocorreram em virtude do AvaDoc (questão 5), acredita-se que o problema está atrelado a pouca familiaridade do usuário com as ferramentas de origem dos documentos. Após o auxílio no uso da ferramenta de origem para realização dos ajustes finais indicados pela recomendações do AvaDoc, verificouse que os documentos gerados apresentavam a acessibilidade necessária para ser, então, disponibilizados para os aprendizes. Os documentos foram utilizados por 8 aprendizes e a avaliação final por parte dos educadores é de que o uso da ferramenta tornou mais simples o processo de incluir acessibilidade em documentos digitais (questão 4), promovendo impactos positivos no processo de ensino e aprendizagem (questões 6 e 8). A Figura 7 apresenta as avaliações realizadas.

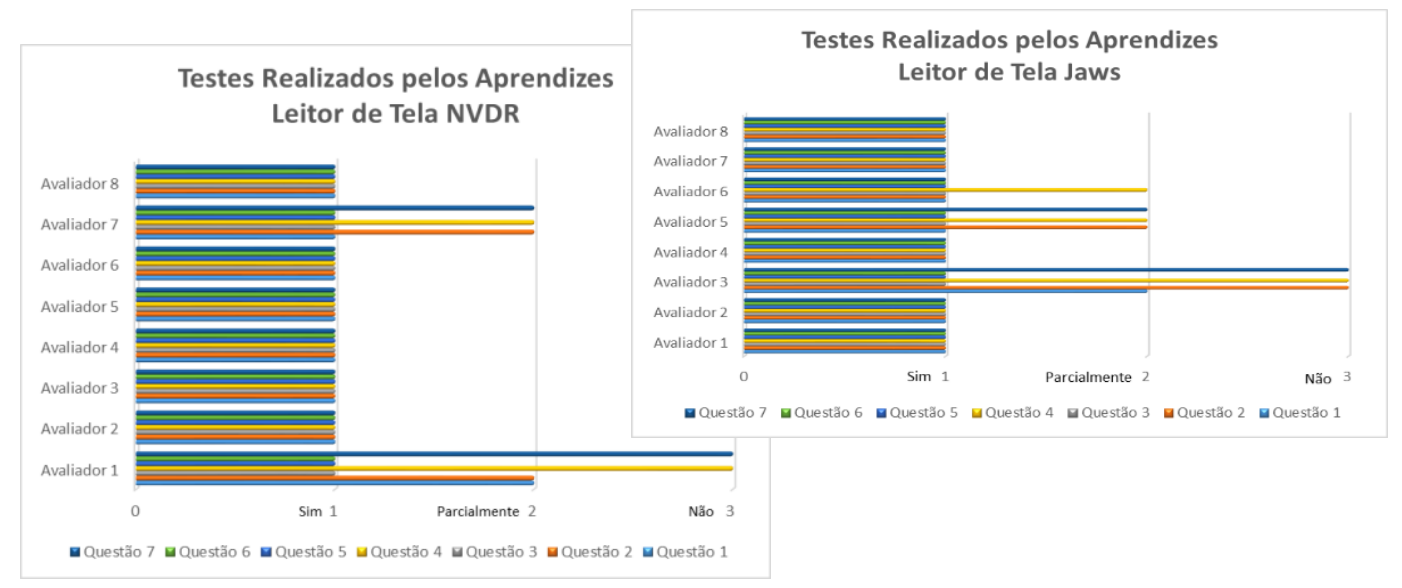

Figura 7. Avaliação do AvaDoc realizada pelos aprendizes

O processo também contou com a colaboração de 8 aprendizes com deficiência visual para avaliar o quanto o documento gerado atendia aos requisitos de acessibilidade. Para isso os documentos resultantes da primeira fase de avaliação foram disponibilizados para utilização por parte dos aprendizes. Para fins de avaliação 
utilizou-se os leitores JAWS R.11/R12 e NVDA R.2010.1. Com base nos gráficos apresentados constata-se que a grande maioria dos avaliadores apresentaram facilidade na leitura dos materiais adaptados (questões 5 e 6), sendo possível navegar por todo o documento, fazendo uso apenas do teclado (questão 1) e localizar as diferentes partes do documento (questão 2). Os problemas encontrados referem-se a pouca familiaridade de alguns usuários quanto ao leitor utilizado (questões 3, 4 e 7). Esta avaliação ressalta o fato de que, mesmo sendo de grande importância a produção de materiais acessíveis para a inclusão da pessoa com deficiência, isso pode não ser o suficiente, pois cada aprendiz pode enfrentar dificuldades com a própria Tecnologia Assistiva que utiliza.

\section{Considerações Finais}

Segundo as leis e normas atuais, aprendizes com deficiência devem ser inseridos na sala de aula do ensino regular. Com isso educadores estão enfrentando dificuldades em adaptar os seus materiais, já elaborados e utilizados na sala de aula, a fim de prover a acessibilidade necessária para que os mesmos possam ser utilizados por todos.

Diante disso, o AvADoc emerge de uma demanda atual relacionada a produção e disponibilização de conteúdos digitais acessíveis, que tem desafiado, sobretudo, educadores. De fato, é fundamental que se criem hábitos de produção de conteúdos acessíveis e corretamente estruturados entre a comunidade acadêmica permitindo, sobretudo, a inclusão de aprendizes com deficiência.

Neste cenário, o avaliador proposto pretende implementar as linhas orientadoras para a criação de documentos acessíveis servindo como um guia, transmitindo recomendações referidas nas diretrizes WCAG 2.0, numa perspectiva operacional e aplicadas aos documentos gerados nos formatos mais comuns. É relevante mencionar que a ferramenta é considerada como um recurso de apoio ao processo de ensino uma vez que apresenta como grande diferencial a possibilidade de produção de material pedagógico acessível. No entanto, quando se fala em tecnologias e recursos que auxiliam o processo de ensino, deve-se lembrar que eles não são recursos que magicamente farão o aluno superar suas dificuldades. Qualquer que seja o auxílio pensado, sempre passa pela percepção que o educador tem sobre as dificuldades e possibilidades de seu aprendiz. O auxílio só faz sentido a partir desta relação.

Além disso, o cumprimento das recomendações propostas pela ferramenta não significa que o acesso à informação fica garantido a todos. Cada aprendiz poderá ter dificuldades específicas, intrínsecas à deficiência ou à tecnologia de apoio que utiliza. Nem todos os utilizadores de tecnologias de apoio (TA) têm a mesma aptidão e experiência, assim como nem toda a TA similar tem o mesmo desempenho [Francisco e Sousa 2011]. Em suma, pretende-se desenvolver uma ferramenta que auxilie, de fato, a interação entre professor-aprendiz no processo de ensino-aprendizagem e que possam ser reconhecidas como uma tecnologia que promova oportunidades de inclusão digital.

O AvaDoc destina-se a todos os públicos que produzem ou disponibilizam conteúdos digitais, sobretudo à docentes, formadores, estudantes e pessoal técnico e administrativo. As avaliações realizadas até o momento demonstram que a ferramenta provê facilidade de aprendizagem e uso, indo de encontro aos princípios básicos de usabilidade, e fornece recomendações adequadas para a concepção de documentos com vistas a torna-los acessíveis. Como perspectiva de continuidade prevê-se a inclusão de novas regras de acessibilidade ao AvADoc, tornando-o mais robusto. 
Por fim, agradecimentos especiais a Secretaria de Ciência, Inovação e Desenvolvimento Tecnológico do Estado do Rio Grande do Sul que, por meio do Programa de Apoio à Polos Tecnológicos, apoia a realização das pesquisas relacionadas a este projeto.

\section{Referências}

ASES - Avaliador e Simulador de Acessibilidade de Sítios. Disponível em $<$ http://www.governoeletronico.gov.br/acoes-e-projetos/e-MAG/ases-avaliador-esimulador-de-acessibilidade-sitios>. Acesso em 08/03/2014.

Conversor Demo of Databeam. Word Component. Versão gratuita disponível em < http://www.duodimension.com/component_convert_word_doc_document_html_net /word_document_doc_html_download.aspx>. Último acesso em 08/08/2013.

Cusin, Cesar Augusto; VIDOTTI. Silvana A. B. G. Acessibilidade em Ambientes Informacionais Digitais. DataGramaZero - Revista de Informação - v.14 n.1. 2013.

DaSilva. Avaliador de Acessibilidade em Português para Web Sites. Disponível em <http://www.dasilva.org.br/?itemid=10>. Último acesso em: 08/08/2013.

Deitel, H. M. Java, como programar. Porto Alegre: Bookman. 2003.

Defendi, Edson Luiz. O livro, a leitura e a pessoa com deficiência visual. São Paulo. 2011.

Falkofske, J. (2008). Making Your Word Documents Accessible. Minnesota State Colleges and Universities System - St. Cloud Technical College. https://sctc.ims.mnscu.edu/shared/FacultyTutorials/TutorialVideos/MakingYourWor dDocumentsAccessible.pdf

Francisco, Manuela. Sousa, Norberto. Guia para produção de conteúdos digitais acessíveis. Edição EU4ALL. 2011.

HERA. Ferramenta para Rever a Acessibilidade das Páginas Web. Disponível em <http://www.sidar.org/hera/index.php.pt>. Ultimo acesso em: 08/08/2013.

OFFICE. Recursos de Acessibilidade. Disponível em <http://office.microsoft.com/ptbr/sharepoint-server-help/recursos-de-acessibilidade-HA010369390.aspx\#top>. Último acesso em: 08/08/2013.

Salton, Bruna Poletto. Criação de Documentos Digitais Acessíveis. Soluções acessíveis: experiências inclusivas no IFRS. Porto Alegre: Companhia Rio-grandense de Artes Gráficas, p.49-69, 2014.

Turró, M. R. (2008). Are PDF documents accessible?. Information Technology and Libraries, v.27 (3) p. 25-43. Disponível em: http://bd.ub.es/pub/ribera/materials/PDFAccessibleI TAL.doc

WCAG. Diretrizes de Acessibilidade para o Conteúdo da Web. Disponível em <http://www.acessibilidade.gov.pt/w3/TR/WCAG20/>. Ultimo acesso em: $08 / 03 / 2014$

W3C. User Agent Accessibility Guidelines (UAAG). Disponível em http://www.w3.org/WAI/intro/uaag.php. Ùltimo acesso em: 05/05/2014. 